\title{
Long non-coding RNA Rian protects against experimental bronchopulmonary dysplasia by sponging miR-421
}

\author{
XIFENG TAO, YAFEI FANG and CHEN HUO \\ Department of Pediatrics, The First People's Hospital of Lianyungang, Lianyungang, Jiangsu 222000, P.R. China
}

Received November 18, 2020; Accepted March 18, 2021

DOI: $10.3892 / \mathrm{etm} .2021 .10213$

\begin{abstract}
Bronchopulmonary dysplasia (BPD) is a frequent complication characterized by accelerated lung alveolarization in newborns. Long non-coding RNAs (lncRNAs) and microRNAs (miRs) are regarded as essential regulators in various diseases, including BPD. However, the detailed mechanism of the functions of RNA imprinted and accumulated in nucleus (Rian) lncRNA in the progression of BPD have remained elusive. The aim of the present study was to illustrate the interaction between miR-421 and Rian in BPD models and MLE-12 cells. The ability of Rian to protect neonatal lungs from hyperoxia-induced lung damage was examined. A mouse model of BPD and a hyperoxia-stimulated MLE-12 cell damage model were generated and treated with specific plasmid/mimics for the overexpression of Rian/miR-421. The interaction between miR-421 and Rian was predicted and verified using StarBase and a dual-luciferase reporter assay, respectively. The expression levels of miR-421 or Rian in both tissues and the MLE-12 alveolar epithelial cell line were assessed using reverse transcription-quantitative (RT-q)PCR. As parameters of alveolarization, the mean linear intercept (MLI), radial alveolar count (RAC) and the lung weight/body weight (LW/BW) ratio were measured. Furthermore, RT-qPCR was used to measure mRNA levels of pro-inflammatory cytokines (TNF- $\alpha$, IL-6 and IL-1 $\beta$ ) in the lung tissue of mice, and ELISAs were performed to determine the levels of pro-inflammatory cytokines (TNF- $\alpha$, IL-6 and IL-1 $\beta$ ) in the supernatant of MLE-12 cells. Cell growth and apoptosis were evaluated using an MTT assay and flow cytometry, respectively. Furthermore, caspase-3 activity was assessed using a caspase-3 activity detection kit. Prediction with StarBase and the dual-luciferase reporter assay revealed that miR-421 directly targeted Rian. RT-qPCR analysis
\end{abstract}

Correspondence to: Dr Yafei Fang, Department of Pediatrics, The First People's Hospital of Lianyungang, 182 Tongguan North Road, Lianyungang, Jiangsu 222000, P.R. China

E-mail: fangyf201112@163.com

Key words: long non-coding RNA, RNA imprinted and accumulated in nucleus, microRNA-421, experimental bronchopulmonary dysplasia confirmed that Rian was downregulated and miR-421 was upregulated in lung tissues of the mouse model of BPD and in hyperoxia-induced MLE-12 cells. However, the expression of miR-421 was decreased by Rian-overexpression, an effect that was reversed by miR-421 mimics. In addition, BPD was alleviated by Rian-plasmid, as confirmed by the enhanced RAC and reduced MLI and LW/BW ratio. The present results also indicated that Rian-plasmid inhibited the secretion of pro-inflammatory cytokines (TNF- $\alpha$, IL-6 and IL-1 $\beta$ ) in BPD mouse serum and hyperoxia-induced MLE-12 cells. In addition, Rian-plasmid eliminated the effect of hyperoxia to inhibit cell viability and induce apoptosis in MLE-12 cells. However, all of these effects of Rian were markedly reversed by miR-421 mimics. The present results indicated that Rian may attenuate hyperoxic damage in neonatal lungs and may serve as a novel molecular target for BPD treatment.

\section{Introduction}

Bronchopulmonary dysplasia (BPD), a serious complication commonly occurring in immature infants, is characterized by arrested alveolarization of the lungs, oxygen toxicity and pulmonary injury $(1,2)$. Studies have indicated that BPD may lead to lung injury, neurodevelopmental sequelae and even disability, resulting in a high economic burden on families (3-5). Hyperoxemia is the major cause of BPD, which is able to exacerbate the damage of alveolar epithelium and endothelial cells (6). Due to perinatal infection and inflammation, immature breathing and oxidative stress, preterm infants are more susceptible to hyperoxia-induced BPD (7-9). In rat models, hyperoxia induction may cause alveolar septation suppression and terminal air space enhancement, which is similar to observations in human BPD $(10,11)$. Hu et al $(12)$ confirmed that microRNA-29a (miRNA/miR-29a) inhibitor was able to relieve hyperoxia-stimulated BPD in neonatal mice through the upregulation of GRB2-associated binding protein 1. At present, effective postnatal treatment strategies for BPD are limited and the major therapeutic methods are mechanical ventilation and drug therapy. In addition, the early identification by nursing staff and active perinatal care in infants with low birth weight and BPD may reduce patient deterioration and improve patient survival (13). Therefore, identifying and seeking effective treatment for BPD is urgent.

Long non-coding RNAs (lncRNAs), a class of non-coding RNA of $>200$ nucleotides in length, have been indicated to 
be associated with various biological processes, including cell proliferation, apoptosis and genomic stability (14). RNA imprinted and accumulated in nucleus (Rian) is an lncRNA with a parent-specific expression, which is located on mouse chromosome 12 and includes three long transcripts: Irm, Meg8 and AB063319 (15,16). A large number of studies have revealed that Rian is a vital regulator of multiple biological processes $(15,17,18)$. For instance, Yao et al (17) indicated that the upregulation of IncRNA Rian suppressed cell apoptosis from cerebral ischemia-reperfusion injury through Rian/miR-144-3p/GATA binding protein 3 signaling.

miRNAs are short non-coding RNAs of 20-22 nucleotides in length, which control gene expression and may also be involved in cancer progression (19). Considerable evidence has proven that miRNAs have vital roles in the pathogenesis of diseases and are considered potential targets for disease therapies. Dupont et al (20) revealed the roles of miRNAs in the transmission of obesity-related metabolic diseases induced by paternal diet. However, the latent mechanisms of miRNAs acting in human diseases remain to be further explored. miR-421 has been confirmed to be upregulated in multiple diseases, including in BPD in mice (21). miR-421 has been indicated to promote the occurrence of osteosarcoma by regulating the expression of MCP induced protein 1 (22). Besides, previous studies have revealed that miR-421 regulates cell growth, migration and apoptosis in several types of cancer by sponging different genes, including ATM serine/threonine kinase and forkhead box O4 $(23,24)$. Moreover, a study has shown that miR-421 is highly expressed in BPD mice, and miR-421 inhibition decreases bronchopulmonary dysplasia in a mouse model by targeting Fgf10 (25). However, the exact functions of miR-421 in BPD have not yet been fully elucidated.

In a bioinformatics analysis, a direct binding site was identified between Rian and miR-421. It was therefore hypothesized that Rian may have an important role in BPD by regulating miR-421. The results of the present study suggested that Rian was downregulated in lung tissues of a rat model of BPD and that Rian-overexpression vector relieved BPD and reduced inflammatory response in BPD rats. In addition, Rian-plasmid was indicated to protect alveolar epithelial cells from hyperoxia-induced damage. However, all of these effects were reversed by miR-421 mimics. The present study suggested that Rian/miR-421 may serve as a novel biomarker for the diagnosis of BPD and as a target for treatment.

\section{Materials and methods}

Animals and BPD model establishment. A total of 60 mice were provided by the Experimental Animal Center of Shanghai and kept under standard conditions $\left(22 \pm 1^{\circ} \mathrm{C}, 55 \pm 5 \%\right.$ humidity, 12-h light/dark cycle) with free access to food and water. All animal operations were in accordance with the National Institutes of Health Guide for the Care and Use of Laboratory Animals. In addition, the present study was approved by the Animal Ethics Committee of the Experimental Animal Center of The First People's Hospital of Lianyungang (Lianyungang, China).

The establishment of the mouse model of BPD was performed as previously described (26). In brief, the neonatal mice (age, $<12 \mathrm{~h}$ ) were stimulated with hyperoxia $\left(85 \% \mathrm{O}_{2}\right)$ or kept in normal room air $\left(21 \% \mathrm{O}_{2}\right)$ for 7 days. The mice were randomly divided into six groups $(\mathrm{n}=10)$ : The control, model, model + control-plasmid (empty vector pcDNA3.1), model + Rian-plasmid (Rian sequence was synthesized based on the Rian sequence and then sub-cloned into the pcDNA3.1 vector; Shanghai GeneChem Co.,Ltd.), model + Rian-plasmid + mimics control (5'-UUCUCCGAACGUGUCACGUTT-3'; RiboBio Co, Ltd.) and the model + Rian-plasmid + miR-421 mimics (5'-AUCAACAGACAUUAAUUGGGCGC-3'; RiboBio Co, Ltd.) group. Mice in the control-plasmid, Rian-plasmid, Rian-plasmid + mimics control and Rian-plasmid + miR-421 mimics groups were intraperitoneally injected once every other day with plasmid/mimics on postpartum days 2-14. Saline $(0.9 \% \mathrm{NaCl})$ was injected into control and model group mice. On postpartum day 14 , the mice were anesthetized with sodium pentobarbital $(50 \mathrm{mg} / \mathrm{kg}$; i.p.) and then sacrificed by cervical dislocation (death was verified by cardiac and respiratory arrest) for lung tissue and blood collection.

Mean linear intercept (MLI) and radial alveolar count (RAC) were calculated in reference to a previous study (4). Furthermore, the lung weight/body weight (LW/BW) ratio of mice was calculated as previously described (4).

Cell culture and treatment. The MLE-12 cells were purchased from the American Type Culture Collection and grown in Hites medium (Cytiva) with 15\% FBS (Gibco; Thermo Fisher Scientific, Inc.) and $100 \mathrm{U} / \mathrm{ml}$ penicillin and streptomycin (Thermo Fisher Scientific, Inc.). The cells were cultivated in an incubator with $5 \% \mathrm{CO}_{2}$ at $37^{\circ} \mathrm{C}$. The MLE-12 cells were then incubated in 12-well plates $\left(5 \times 10^{4}\right.$ cells per well) for $24 \mathrm{~h}$ and stimulated with Hites medium supplemented with $0.1 \%$ FBS for $6 \mathrm{~h}$. Next, the MLE-12 cells were exposed to room air or hyperoxia ( $85 \%$ oxygen) for $6 \mathrm{~h}$ and treated with or without $\mathrm{plasmid} / \mathrm{mimics}$ for $24 \mathrm{~h}$.

Reverse transcription-quantitative $(R T-q) P C R$ analysis. The isolation of RNA from the lung tissue of mice with BPD and MLE-12 cells was performed using TRIzol ${ }^{\circledR}$ reagent (Thermo Fisher Scientific, Inc.) following the manufacturer's protocol. Total RNA was then reverse transcribed into cDNA by using the HiScript ${ }^{\mathrm{TM}}$ II qRT SuperMix (Vazyme Biotech Co., Ltd.). RT conditions were as follows: $25^{\circ} \mathrm{C}$ for $5 \mathrm{~min}, 42^{\circ} \mathrm{C}$ for $60 \mathrm{~min}$ and $80^{\circ} \mathrm{C}$ for $2 \mathrm{~min}$. The expression of Rian and miR-421 was detected using SYBR Premix Ex-Taq (Takara Bio, Inc.). The thermocycling conditions were as follows: Initial denaturation at $95^{\circ} \mathrm{C}$ for $5 \mathrm{~min} ; 38$ cycles of denaturation at $95^{\circ} \mathrm{C}$ for $10 \mathrm{sec}$, annealing at $60^{\circ} \mathrm{C}$ for $20 \mathrm{sec}$ and extension at $72^{\circ} \mathrm{C}$ for $30 \mathrm{sec}$. Primers were obtained from Sangon Biotech Co., Ltd. as follows: GAPDH, forward, 5'-CTTTGGTATCGTGGA AGGACTC-3' and reverse, 5'-GTAGAGGCAGGGATGATG TTCT-3'; U6, forward, 5'-GCTTCGGCAGCACATATACTA AAAT-3' and reverse, 5'-CGCTTCACGAATTTGCGTGTC AT-3'; lncRNA Rian, forward, 5'-CTGTTGTGCCCTCCC TGGATG-3' and reverse, 5'-CCAGCTAGGCTGTGTAAA TCATC-3'; miR-421, forward, 5'-GTCGCGCGGGUUAAU GCCTC-3' and reverse, 5'-GGACATUAGUUGUCUGUA AATAG-3'; TNF- $\alpha$, forward, 5'-CCGGGAGAAGAGGGA TAGCTT-3' and reverse, 5'-TCGGACAGTCACTCACCA AGT-3'; IL-6, forward: 5'-TAGTCCTTCCTACCCCAATTT 
CC-3' and reverse, 5'-TTGGTCCTTAGCCACTCCTTC-3'; IL-1 $\beta$, forward: 5'-GAAATGCCACCTTTTGACAGTG-3' and reverse, CTGGATGCTCTCATCAGGACA-3'. The reactions were run in triplicate using the ABI PRISM 7900 sequence detection system (Thermo Fisher Scientific, Inc.). Target gene expression was calculated with the $2^{-\Delta \Delta C q}$ method (27).

Dual-luciferase reporter assay. Bioinformatics software (StarBase version 2.0; http://starbase.sysu.edu. $\mathrm{cn} /$ starbase2/index.php) was used to predict potential target genes of Rian using the 'miRNA-lncRNA' functions as described previously (28). The binding sites between miR-421 and Rian were observed. The Rian-3'untranslated region (UTR), which contains the miR-421 binding site, or the mutated (MUT) target site, was synthesized via genomic PCR (29) from total RNA preps extracted from MLE-12 cells and cloned into pMIR vectors (Thermo Fisher Scientific, Inc.) to construct the reporter vector Rian-wild-type (Rian-WT) or Rian-MUT-type (Rian-MUT). To point-mutate the miR-421 binding domain in the 3'UTR of Rian, a QuikChange Site-Directed Mutagenesis kit (Stratagene; Agilent Technologies, Inc.) was used according to the manufacturer's instructions. The 293T cells (American Tissue Culture Collection) were transfected with Rian WT or MUT plasmid combined with miR-421 mimics or mimics control using Lipofectamine ${ }^{\circledR} 2000$ (Thermo Fisher Scientific, Inc.) following the manufacturer's protocol. The luciferase activity was determined using a Dual-Luciferase Reporter Assay system (Promega Corp.) and normalized to Renilla activity.

ELISA. Inflammatory factors (TNF- $\alpha$, IL-6 and IL-1 $\beta$ ) in the supernatant of hyperoxia-induced MLE-12 cells were evaluated using ELISA kits (cat. nos. PT512, PI326 and PI301; BioLegend, Inc.) following the manufacturer's protocol. The optical density (OD) value in each well was detected at $450 \mathrm{~nm}$ following the manufacturer's protocol of the ELISA kits.

Cell transfection. The IncRNA sequence for Rian was synthesized based on the Rian sequence and then sub-cloned into the pcDNA3.1 vector (Shanghai GeneChem Co., Ltd.) to obtain Rian-plasmid. The empty pcDNA3.1 vector was used as a control (control-plasmid; Shanghai GeneChem Co., Ltd.). MLE-12 cells were cultured in room air or under hyperoxia for $6 \mathrm{~h}$. Subsequently, $100 \mathrm{ng}$ control-plasmid, 100 ng Rian-plasmid, $100 \mathrm{nM}$ mimics control (5'-UUCUCC GAACGUGUCACGUTT-3'; RiboBio Co, Ltd.) or $100 \mathrm{nM}$ miR-421 mimics (5'-AUCAACAGACAUUAAUUGGGC GC-3'; RiboBio Co, Ltd.) were transfected into MLE-12 cells using Lipofectamine ${ }^{\circledR} 2000$ (Thermo Fisher Scientific, Inc.) for $24 \mathrm{~h}$, following the manufacturer's protocol. At $24 \mathrm{~h}$ after cell transfection, subsequent experimentation was performed and the overexpression efficiency was determined using RT-qPCR.

MTT assay. Following transfection for $24 \mathrm{~h}$, MLE-12 cells were seeded into 96 -well plates $\left(1 \times 10^{4}\right.$ cells/well; $100 \mu 1$ suspension/well) and incubated for $24 \mathrm{~h}$ at $37^{\circ} \mathrm{C}$. Cells were then exposed to MTT ( $10 \mu \mathrm{l}$ of $5 \mathrm{mg} / \mathrm{ml}$ added per well) for $4 \mathrm{~h}$ at $37^{\circ} \mathrm{C}$. Following treatment, the solution was removed and $100 \mu \mathrm{l}$ DMSO was added to each well to dissolve the formazan product. Finally, the OD at a wavelength of $570 \mathrm{~nm}$ was

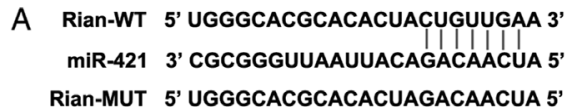

Rian-MUT 5' UGGGCACGCACACUAGACAACUA 5

B

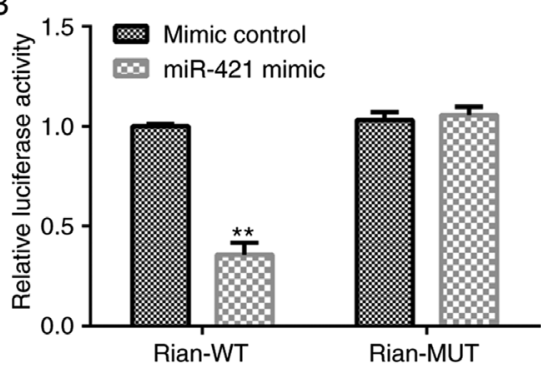

Figure 1. miR-421 is a direct target of long non-coding RNA Rian. (A) Schematic of miR-421 binding site in the 3'-untranslated region of Rian. (B) Dual-luciferase reporter assay with the combinations of miR-421 mimics/control and WT/MUT Rian confirmed the direct targeting interaction. ${ }^{* *} \mathrm{P}<0.01$ vs. mimics control group. miR/miRNA, microRNA; WT, wild-type; MUT, mutant; Rian, RNA imprinted and accumulated in nucleus.

assessed using a multifunctional plate reader (BioTek China) after 15 min of vibration mixing, following the manufacturer's protocols.

Flow cytometry. Following transfection for $24 \mathrm{~h}$, MLE-12 cell apoptosis was determined with an Annexin V-FITC/PI apoptosis detection kit (BD Biosciences), according to the manufacturer's protocol. A flow cytometer (BD Biosciences) was used to quantify cell apoptosis and the results were analyzed using CellQuest software (version 5.1; BD Biosciences).

Detection of caspase-3 activity. The caspase-3 activity in MLE-12 cells was assessed using a Caspase-3 Assay kit (cat. no. ab39401; Abcam), following the manufacturer's instructions. In brief, MLE-12 cells were disintegrated with buffer solution. Lysate was obtained and centrifuged at $4^{\circ} \mathrm{C}$ for $10 \mathrm{~min}$ at $1,500 \mathrm{xg}$. The supernatant was then treated with caspase- 3 reagent, followed by incubation at $37^{\circ} \mathrm{C}$ for $2 \mathrm{~h}$. Following treatment, a microplate reader (BMG Labtech $\mathrm{GmbH}$ ) was used to measure the OD indicative of caspase-3 activity at $405 \mathrm{~nm}$.

Statistical analysis. Statistical analysis was performed using SPSS 20.0 (IBM, Corp.). Values are expressed as the mean \pm standard deviation from three independent experiments. Mean differences between two groups were estimated with an unpaired Student's t-test and those among multiple groups by one-way ANOVA followed by Tukey's post-hoc test. $\mathrm{P}<0.05$ was considered to indicate a statistically significant difference.

\section{Results}

miR-421 directly interacts with Rian. First, the potential association between miR-421 and Rian was investigated. The prediction with StarBase indicated that miR-421 was a latent target of Rian (Fig. 1A). Furthermore, the binding site of miR-421 on Rian was confirmed via a dual-luciferase reporter assay. In the luciferase reporter assay, miR-421 mimics significantly decreased the luciferase activity of Rian-WT reporter plasmid, while no changes were observed in the Rian-MUT 

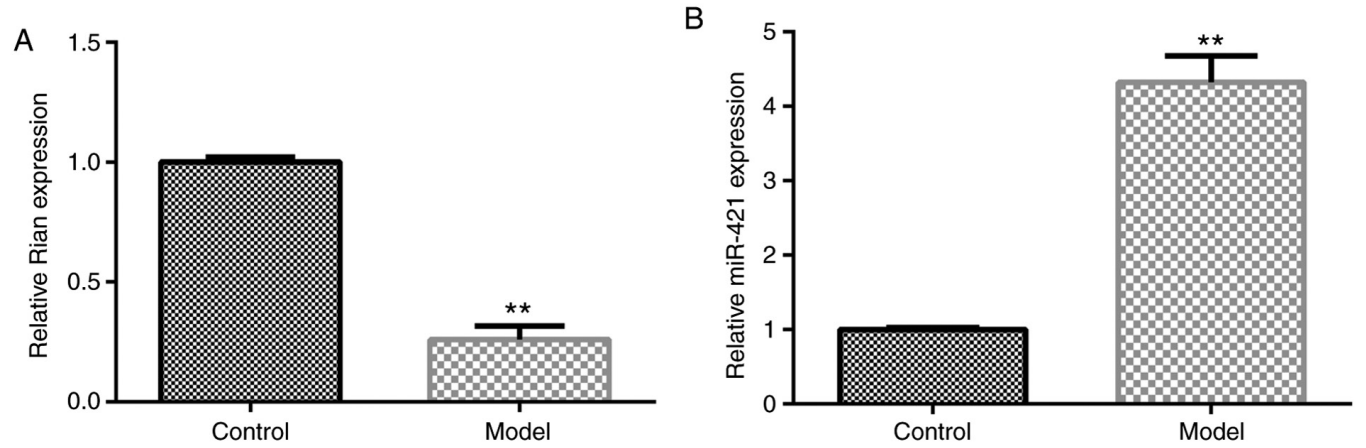

Figure 2. Rian is downregulated and miR-421 upregulated in lung tissues of the mouse model of BPD. Expression of (A) Rian and (B) miR-421 in lung tissues of mice with BPD were assessed using reverse transcription-quantitative PCR. ${ }^{* * *} \mathrm{P}<0.01$ vs. control group. miR, microRNA; BPD, bronchopulmonary dysplasia; Rian, RNA imprinted and accumulated in nucleus.

A

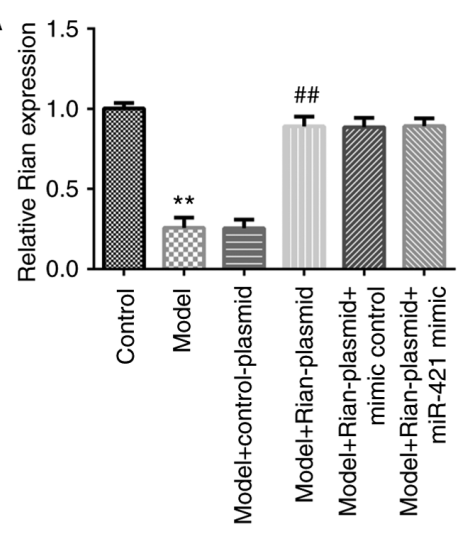

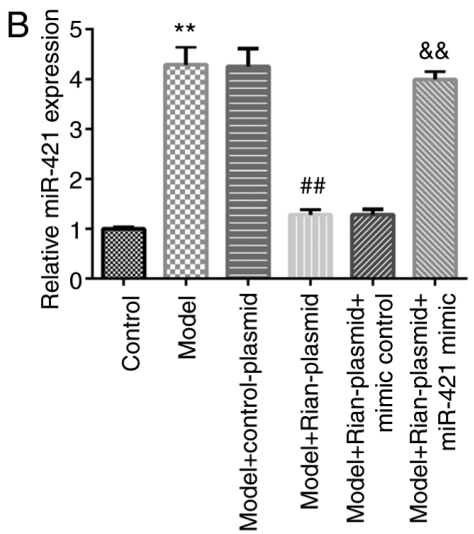
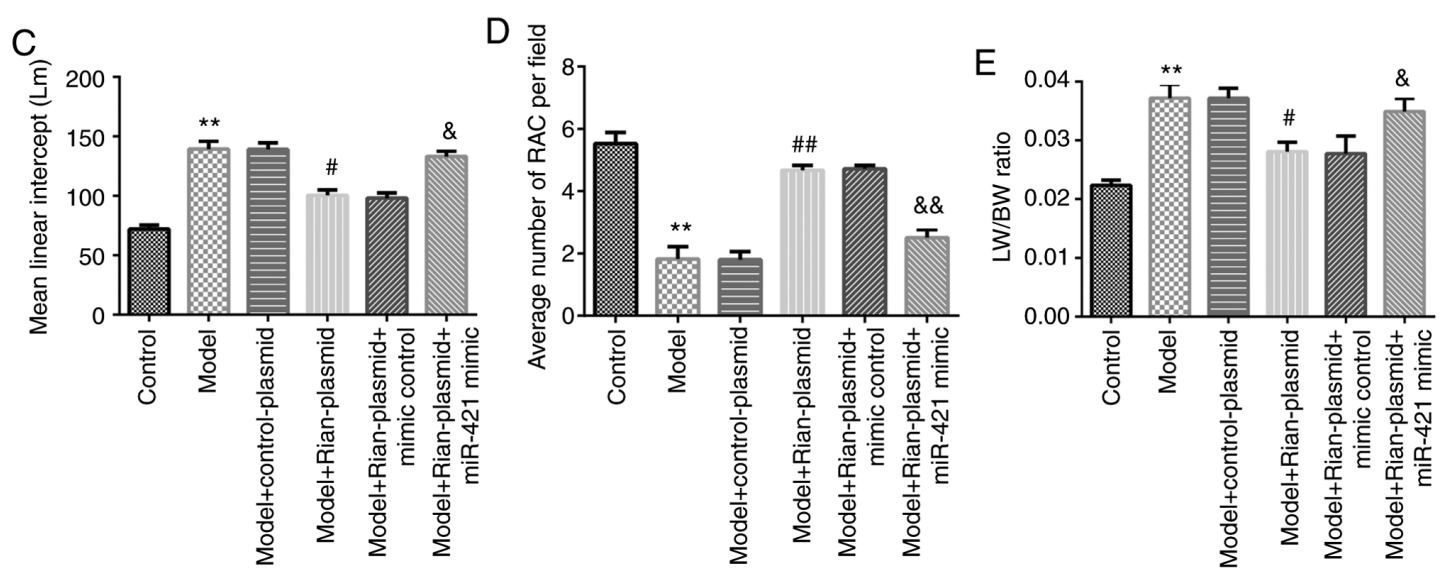

Figure 3. miR-421 mimics reverse the effects of Rian-overexpression vector on the parameters indicative of BPD. Control-plasmid, Rian-plasmid, mimics control or miR-421 mimics were injected into BPD mice. (A and B) Reverse transcription-quantitative PCR analysis of (A) Rian and (B) miR-421 expression in the lung tissue of mice with BPD in different groups. (C-E) Determination of (C) MLI, (D) RAC and (E) LW/BW ratios in different groups. ${ }^{* *} \mathrm{P}<0.01$ vs. Control; ${ }^{*} \mathrm{P}<0.05$ and ${ }^{\# \#} \mathrm{P}<0.01$ vs. Model + control-plasmid group; ${ }^{\circledR} \mathrm{P}<0.05$ and ${ }^{\& \&} \mathrm{P}<0.01$ vs. Model + Rian-plasmid + mimics control group. miR, microRNA; BPD, bronchopulmonary dysplasia; MLI, mean linear intercept; RAC, radial alveolar count; LW/BW, lung weight/body weight; Rian, RNA imprinted and accumulated in nucleus.

luciferase activity $(\mathrm{P}<0.01$; Fig. 1B). These observations suggested that miR-421 directly binds to Rian.

Expression of miR-421 and Rian in the lung tissue of BPD mice. Next, the role of miR-421 or Rian in the progression of BPD was evaluated using RT-qPCR. The results indicated that Rian was notably downregulated in BPD mouse lung tissues as compared to the control $(\mathrm{P}<0.01$; Fig. $2 \mathrm{~A})$. Furthermore, the level of miR-421 was markedly upregulated in the lung tissue of BPD mice as compared to the control ( $\mathrm{P}<0.01$; Fig. 2B). These results demonstrated that miR-421/Rian may have a vital role in the pathogenesis of BPD.

miR-421 mimics abolish the influence of Rian-plasmid on BPD mice. To further reveal the functions of Rian and miR-421 in the development of BPD, control-plasmid, Rian-plasmid, mimics control or miR-421 mimics were injected into BPD mice and the levels of Rian or miR-421 were evaluated using 

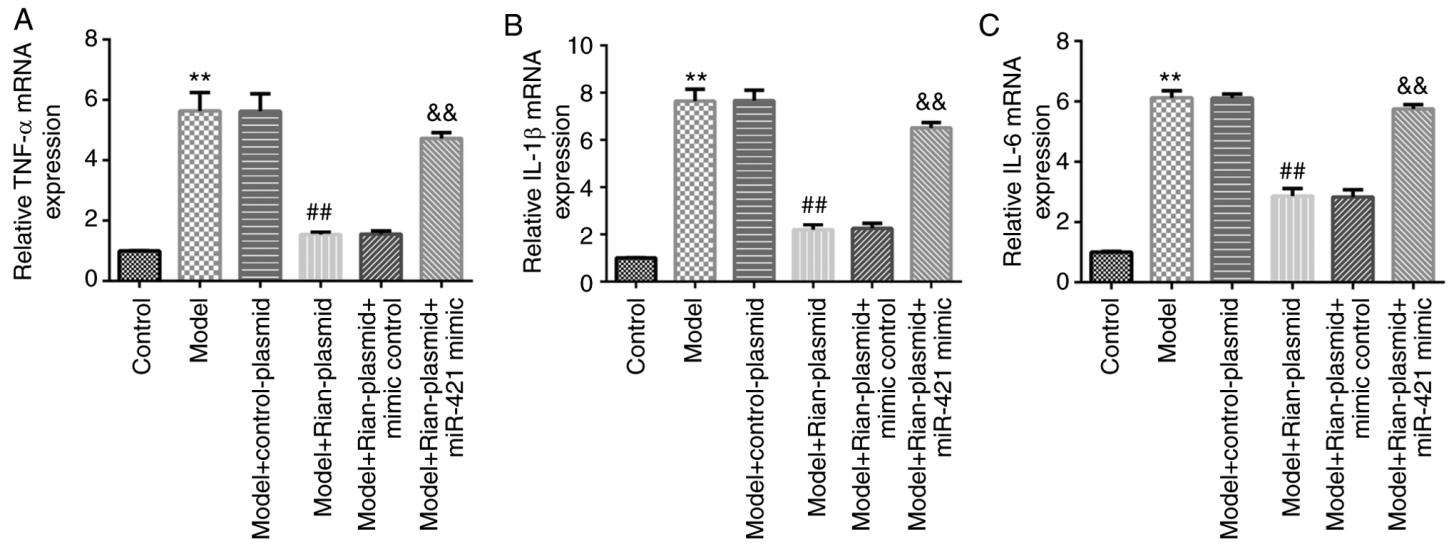

Figure 4. Effects of miR-421 mimics or Rian-plasmid on inflammatory factors in BPD mice. Control-plasmid, Rian-plasmid, mimics control or miR-421 were injected into BPD mice. mRNA levels of (A) TNF- $\alpha$, (B) IL-1 $\beta$ and (C) IL- 6 in the lung tissue of mice were detected using reverse transcription-quantitative PCR. ${ }^{* *} \mathrm{P}<0.01$ vs. Control; ${ }^{\# \#} \mathrm{P}<0.01$ vs. Model + control-plasmid group; ${ }^{\& \&} \mathrm{P}<0.01$ vs. Model + Rian-plasmid + mimics control group. miR, microRNA; $\mathrm{BPD}$, bronchopulmonary dysplasia; Rian, RNA imprinted and accumulated in nucleus.

A

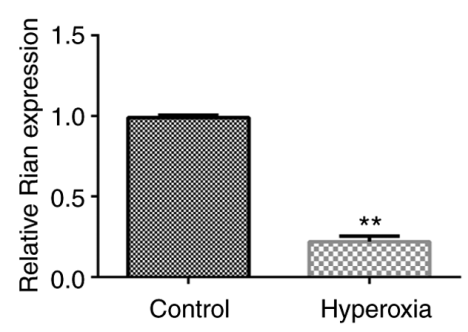

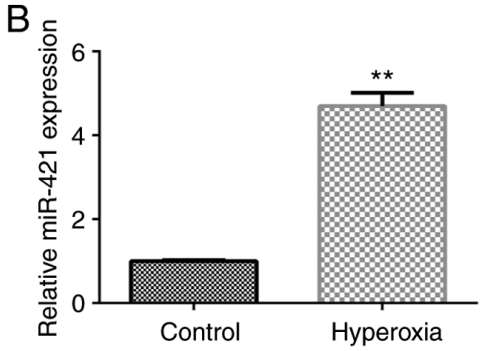

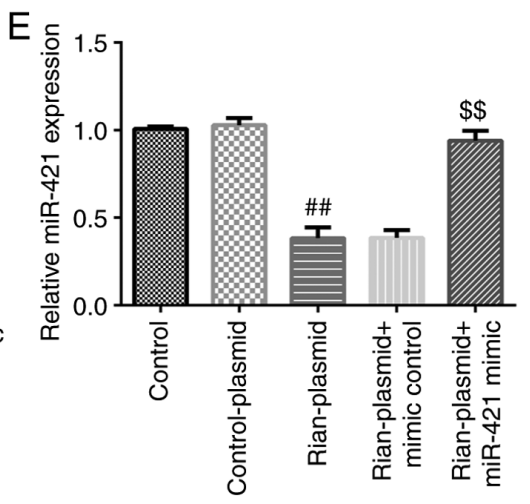

Figure 5. Effects hyperoxia or Rian-plasmid or miR-421 mimics on Rian or miR-421 expression in MLE-12 cells determined by reverse transcription-quantitative PCR. (A) Rian and (B) miR-421 levels in hyperoxia-induced lung cells. (C and D) Confirmation of overexpression of (C) Rian in the Rian-plasmid group and (D) miR-421 in the mimics group compared with the respective control groups. (E) miR-421 expression following transfection Rian-plasmid with or without miR-421 mimics or controls. ${ }^{* *} \mathrm{P}<0.01$ vs. Control; ${ }^{\# \#} \mathrm{P}<0.01$ vs. control-plasmid group; ${ }^{\& \&} \mathrm{P}<0.01 \mathrm{vs}$. mimic control group; ${ }^{\$} \mathrm{P}<0.01 \mathrm{vs}$. Rian-plasmid + mimics control. miR, microRNA; Rian, RNA imprinted and accumulated in nucleus.

RT-qPCR. Rian was indicated to be downregulated in BPD mouse lung tissues as compared with those in the control group, while Rian-plasmid significantly increased Rian expression compared with that in the model + control plasmid group $(\mathrm{P}<0.01)$. No obvious differences in the expression of Rian were observed among the model + Rian-plasmid, model + Rian-plasmid + mimics control and model + Rian-plasmid + miR-421 mimics groups (Fig. 3A). In addition, as presented in Fig. 3B, Rian-plasmid markedly suppressed the level of miR-421 in BPD mouse lung tissues, as compared with that in the control-plasmid group; however, the effect of Rian-plasmid on miR-421 was reversed by miR-421 mimics $(\mathrm{P}<0.01$;
Fig. 3B). Furthermore, quantitative analyses of the RAC and the MLI of mice in different groups were performed to evaluate the hyperoxia-induced lung damage and the LW/BW ratio was calculated as an index of lung injury $(4,30,31)$. An increased MLI ( $\mathrm{P}<0.01$; Fig. 3C), suppressed $\mathrm{RAC}(\mathrm{P}<0.01$; Fig. 3D) and enhanced LW/BW ratio $(\mathrm{P}<0.01$; Fig. $3 \mathrm{E})$ were observed in model mice. As compared with the Model + control-plasmid group, Rian-plasmid significantly decreased the MLI level $(\mathrm{P}<0.05)$, enhanced RAC expression $(\mathrm{P}<0.01)$ and reduced the $\mathrm{LW} / \mathrm{BW}$ ratio $(\mathrm{P}<0.05)$, and these effects were reversed by miR-421 mimics. In conclusion, the present results suggested that Rian exerted its effect in BPD via miR-421. 

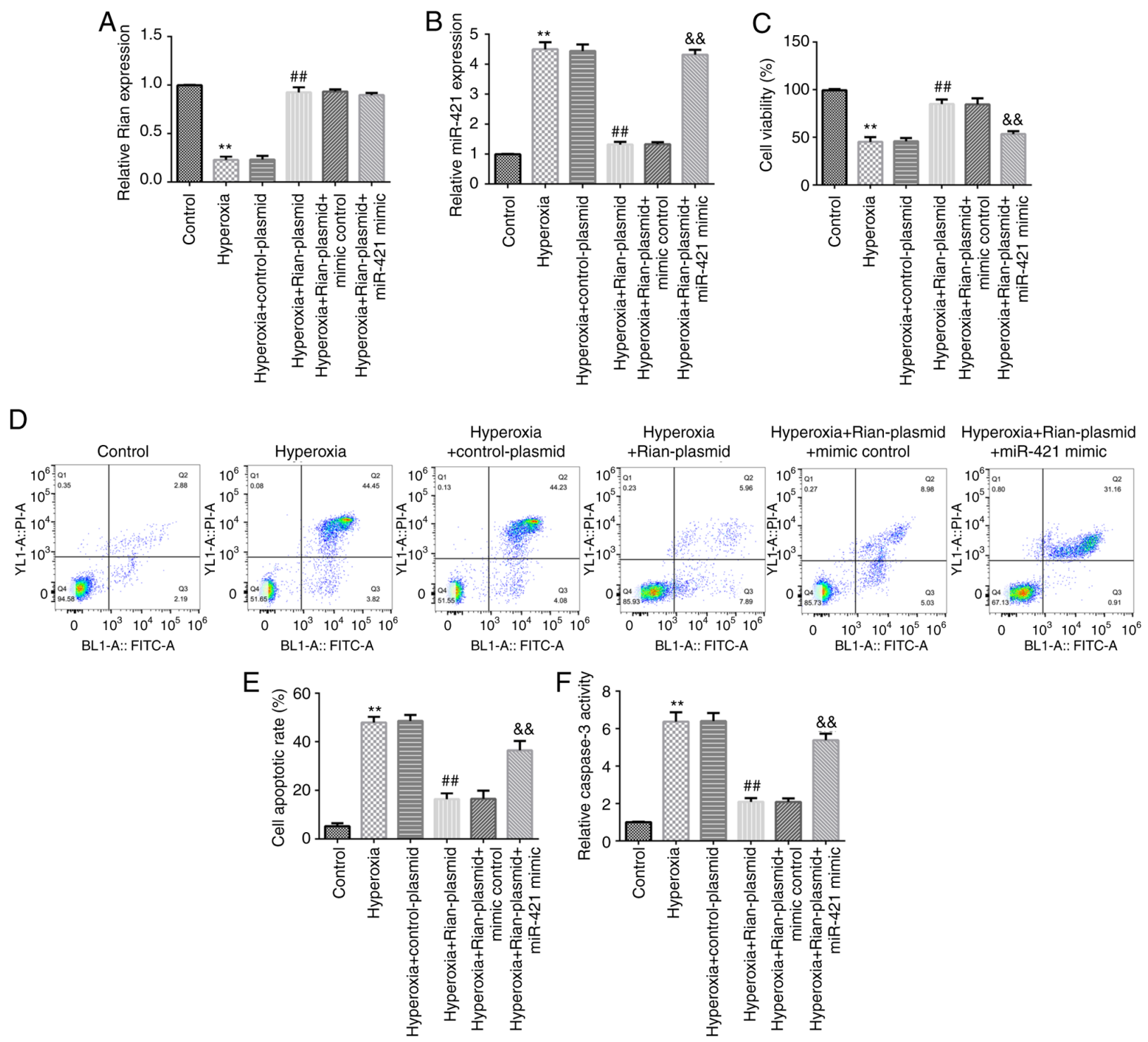

Figure 6. miR-421 mimics reverse the effects of Rian-plasmid on MLE-12 cell viability and apoptosis. Following stimulation (hyperoxia induction) and transfection, cells were divided into six groups: Control, hyperoxia, hyperoxia + control-plasmid, hyperoxia + Rian-plasmid, hyperoxia + Rian-plasmid + mimics control, or hyperoxia + Rian-plasmid + miR-421 mimics groups. Reverse transcription-quantitative PCR of (A) Rian and (B) miR-421 expression in different groups. (C) Cell viability was evaluated with an MTT assay. (D) Apoptotic cells were determined using flow cytometry. (E) Quantitative analysis of apoptotic cells from D. (F) Determination of caspase-3 activity in different groups. ${ }^{* * *} \mathrm{P}<0.01$ vs. Control; ${ }^{\# \#} \mathrm{P}<0.01$ vs. hyperoxia + control-plasmid group; \&\& $\mathrm{P}<0.01$ vs. hyperoxia + Rian-plasmid + mimics control group. miR, microRNA; Rian, RNA imprinted and accumulated in nucleus.

miR-421 mimics reverse the effects of Rian-plasmid on inflammatory response in BPD mice. Given that immunoreaction is a vital marker in the pathophysiological processes of BPD (7), RT-qPCR was performed to examine the levels of inflammatory factors (TNF- $\alpha$, IL- 6 and IL-1 $\beta$ ) in the lung tissue of mice from different groups. The results revealed that TNF- $\alpha$ (Fig. 4A), IL-6 (Fig. 4B) and IL-1 $\beta$ (Fig. 4C) levels were notably elevated in model mouse lung tissues $(\mathrm{P}<0.01)$. In addition, in the Model + Rian-plasmid group, the levels of TNF- $\alpha$, IL- 6 and IL-1 $\beta$ were markedly inhibited as compared with those in the Model + control-plasmid group $(\mathrm{P}<0.01)$. However, these inhibitions were reversed following miR-421 mimics treatment.

Effects of Rian-plasmid and miR-421 mimics on Rian or miR-421 expression in hyperoxia-induced lung cells. Furthermore, the expression of Rian and miR-421 in hyperoxia-induced lung cells were determined. RT-qPCR analysis indicated that Rian was downregulated and miR-421 was upregulated in hyperoxia-induced lung cells (Fig. 5A and B). Then, to determine whether Rian could regulate miR-421 level, MLE-12 cells were transfected with control-plasmid, Rian-plasmid, mimics control or miR-421 mimics for $24 \mathrm{~h}$. Results indicated that as compared with that in the control-plasmid group, the level of Rian was clearly increased in the Rian-plasmid group ( $\mathrm{P}<0.01$; Fig. 5C). miR-421 was determined to be overexpressed in miR-421 mimics-transfected cells, as compared with the mimics control group ( $\mathrm{P}<0.01$; Fig. 5D). Furthermore, Rian-plasmid significantly decreased miR-421 levels and this inhibition was reversed by miR-421 mimics $(\mathrm{P}<0.01$; Fig. $5 \mathrm{E})$. Based on these in vitro results, it was hypothesized that Rian may be able to protect against hyperoxia-induced lung damage, which was then further assessed. 

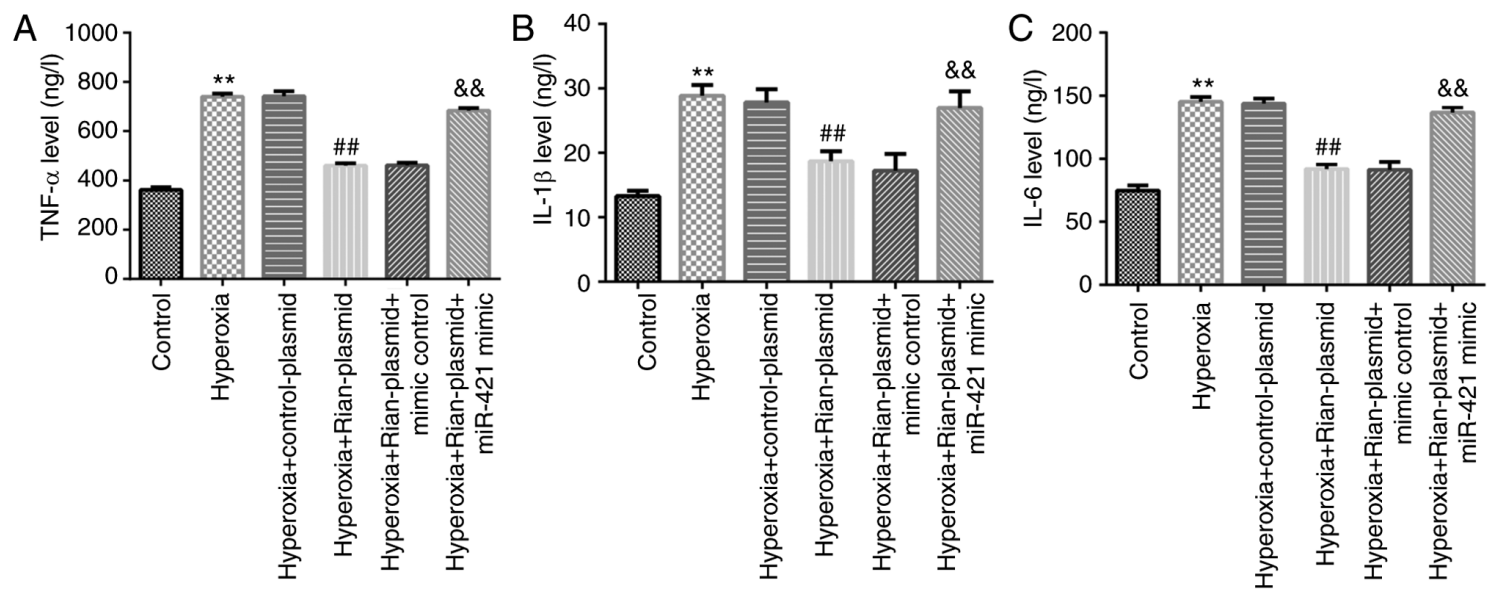

Figure 7. miR-421 mimics abolish the effects of Rian-plasmid on inflammatory response in hyperoxia-induced MLE-12 cells. Following hyperoxia induction and transfection, cells were divided into six groups: Control, hyperoxia, hyperoxia + control-plasmid, hyperoxia + Rian-plasmid, hyperoxia + Rian-plasmid + mimics control, or hyperoxia + Rian-plasmid + miR-421 mimics groups. The expression of (A) TNF- $\alpha$, (B) IL-1 $\beta$ and (C) IL-6 and in MLE-12 cells was evaluated via ELISA. ${ }^{* *} \mathrm{P}<0.01$ vs. Control; ${ }^{\# \#} \mathrm{P}<0.01$ vs. hyperoxia + control-plasmid group; \&\& $\mathrm{P}<0.01$ vs. hyperoxia + Rian-plasmid + mimics control group. miR, microRNA; Rian, RNA imprinted and accumulated in nucleus.

miR-421 mimics reverse the effects of Rian-plasmid on MLE-12 cell viability and apoptosis. To further analyze the role of Rian in hyperoxia-induced lung cells, MLE-12 cells were cultured under room air $\left(21 \% \mathrm{O}_{2}\right)$ or hyperoxia $\left(85 \% \mathrm{O}_{2}\right)$ for $6 \mathrm{~h}$. Control-plasmid, Rian-plasmid, mimics control or miR-421 mimics were then transfected into cells for $24 \mathrm{~h}$. Subsequently, the expression of Rian or miR-421 in the different groups was analyzed. The results of the RT-qPCR analysis demonstrated that the level of Rian was markedly decreased in the hyperoxia group in comparison to the control group and markedly increased in the hyperoxia + Rian-plasmid group in comparison to the hyperoxia + control plasmid group. However, no obvious difference in the levels of Rian was observed among the hyperoxia + Rian-plasmid, hyperoxia + Rian-plasmid + mimics control and hyperoxia + Rian-plasmid + miR-421 mimics groups (Fig. 6A). Furthermore, miR-421 was upregulated in the hyperoxia-induced lung cells in comparison with the control group and downregulated in the hyperoxia + Rian-plasmid group as compared with that in the hyperoxia + control plasmid group. However, in comparison with the hyperoxia + Rian-plasmid + mimics control group, miR-421 mimics increased the level of miR-421 in the hyperoxia-induced MLE-12 cells co-transfected with Rian-plasmid (Fig. 6B). Further analysis using the MTT assay and flow cytometry revealed that in the hyperoxia group, cell viability was inhibited (Fig. 6C) and apoptosis was promoted (Fig. 6D and E) in comparison to the control group. Caspase-3 activity was also enhanced in the hyperoxia group compared to the control group (Fig. 6F). Transfection with Rian-plasmid was able to reverse the effects of hyperoxia, which was canceled out by miR-421 mimics. Therefore, the above results indicated that in BPD, lncRNA Rian protected against hyperoxia-induced lung cell injury through targeting miR-421.

miR-421 mimics reverse the effects of Rian-plasmid on inflammatory response in hyperoxia-induced lung cells. Furthermore, in the present study, the molecular mechanisms of action of IncRNA Rian in hyperoxia-induced lung cells were examined. As presented in Fig. 7, the levels of TNF- $\alpha$,
IL-6 and IL-1 $\beta$ were markedly enhanced in hyperoxia-induced lung cells. In addition, as compared with those in the hyperoxia + control-plasmid groups, the TNF- $\alpha$, IL-6 and IL-1 $\beta$ levels were markedly reduced in the hyperoxia + Rian-plasmid group $(\mathrm{P}<0.01)$. However, these effects were abolished by miR-421 mimics. The above results suggested that lncRNA Rian is a crucial regulator in BPD development by sponging miR-421.

\section{Discussion}

BPD is a serious lung disease with a high incidence in newborns. Previous studies have indicated that newborns with BPD frequently have respiratory and neurological diseases $(32,33)$. At present, the major treatments for BPD are mechanical rehabilitation and drug therapy. Despite the fact that various studies have explored therapeutic strategies of BPD $(34,35)$, the pathophysiological mechanisms and pathogenesis of BPD have remained largely elusive. In addition, certain studies have demonstrated that accurate diagnosis of BPD and interdisciplinary care for affected pediatric patients were beneficial for the treatment of BPD $(36,37)$. Therefore, identifying effective therapeutic strategies for BPD has become a key challenge in recent years.

LncRNAs have been indicated to have crucial roles in various diseases. A study by Cai et al (38) demonstrated that lncRNA gallbladder cancer drug resistance-associated lncRNA1 (GBCDRlnc1) induces chemoresistance of gallbladder carcinoma cells by activating autophagy. Several studies have demonstrated that Rian is an important regulator of biological processes $(15,17-19)$. However, the role of lncRNA Rian in BPD has remained to be fully explored. Therefore, the present study was designed to examine the functions of Rian and related mechanisms in lung tissues in BPD.

Studies have confirmed that lncRNAs and miRNAs have vital roles in the pathological mechanisms of BPD. Certain miRNAs were confirmed to be vital regulators in disease development. They may serve as oncogenes or suppressors in different malignancies. For instance, miR-574-3p was 
reported to regulate adrenomedullin to protect premature infants with BPD (39). A study by Wang et al (40) revealed mRNA, lncRNA, circular RNA and miRNA expression patterns in BPD mouse lung tissues. Another study confirmed that miR-421 was upregulated in BPD mice (25). Furthermore, miR-421 upregulation or fibroblast growth factor 10 (FGF10) silencing intensified the progression of BPD, while miR-421 inhibition alleviated bronchopulmonary dysplasia in a mouse model by targeting FGF10 (25). First, based on a bioinformatics prediction with StarBase, a dual-luciferase reporter assay confirmed that lncRNA Rian directly regulated miR-421 by sponging its 3'UTR. Next, BPD mouse models were generated and the miR-421 or Rian levels in the model and control groups were evaluated by RT-qPCR. miR-421 was determined to be upregulated, while Rian was downregulated in BPD mice as compared to the control group, suggesting that miR-421 and Rian were involved in the regulation of BPD. Recent studies have confirmed that the altered expression of certain miRNAs is associated with tumor diagnosis and prognosis $(41,42)$. It was speculated that an altered expression of Rian or miR-421 may obstruct the development of BPD. To confirm the present hypothesis, control-plasmid, Rian-plasmid, mimics control or miR-421 mimics were injected in the peritoneum of the mice every other day between days 2 and 14 after birth. The RT-qPCR results demonstrated that Rian-plasmid prominently increased Rian expression in BPD lung tissues and suppressed the miR-421 levels, while the effects of Rian-plasmid were abolished by miR-421 mimics. In conclusion, the present results suggested that miR-421 mimics interfered with the functions of Rian in BPD mouse lung tissues.

The effects of Rian-plasmid or miR-421 mimics on BPD in mice were also evaluated in the present study. To evaluate the hyperoxia-induced lung histological damage, quantitative analyses of the RAC and the MLI were performed and the LW/BW ratio was determined as an index of lung injury $(4,30,31)$. On day 14 from plasmid/mimics injection, the MLI, RAC and $\mathrm{LW} / \mathrm{BW}$ ratios were evaluated in different groups to assess the injury in BPD mice. The results suggested that Rian-plasmid relieved mouse BPD, as evidenced by enhanced RAC, reduced MLI and LW/BW ratio. However, these effects on the MLI, RAC and LW/BW ratio were reversed by miR-421 mimics. Supplemental oxygen provided to newborns with BPD may cause oxidative stress and cytokine secretion (43). It was previously verified that increased levels of pro-inflammatory factors are associated with BPD progression (44). To further explore the functions of Rian in BPD, pro-inflammatory cytokine secretion in BPD mice was detected after plasmid/mimics treatment. It was observed that Rian-plasmid reduced the expression of TNF- $\alpha$, IL- 6 and IL- $1 \beta$ in BPD mouse serum. Further analysis suggested that these effects were clearly reversed by miR-421 mimics.

Prolonged hyperoxia exposure may result in lung damage through the production of highly destructive oxygen radicals (45). Next, the effect of Rian on hyperoxia-induced lung cell injury in vitro was explored. The RT-qPCR results indicated that miR-421 was significantly overexpressed and Rian was clearly downregulated in hyperoxia-induced MLE-12 cells, as compared to the control. In addition, Rian-plasmid enhanced Rian levels and suppressed miR-421 expression in MLE-12 cells, while this inhibition was reversed by miR-421 mimics. In addition, miR-421 was upregulated in miR-421 mimics-transfected MLE-12 cells. To further understand the functional role of Rian in BPD, its effects on MLE-12-cell viability, apoptosis and inflammatory factor secretion were examined.

Following exposure to room air $\left(21 \% \mathrm{O}_{2}\right)$ or hyperoxia $(85 \%$ $\mathrm{O}_{2}$ ) for $6 \mathrm{~h}, \mathrm{MLE}-12$ cells were transfected with control-plasmid, Rian-plasmid, mimics control or miR-421 mimics for $24 \mathrm{~h}$. Previous studies have demonstrated the vital roles of cell viability and apoptosis in the occurrence of BPD (46). Consistent with previous studies, it was indicated that Rian protects against hyperoxia-induced lung injury, as evidenced by increased cell viability and reduced apoptosis, and these effects were reversed by miR-421 mimics. In addition, the activity of caspase-3 was markedly depressed in Rian-plasmid-induced MLE-12 cells, and this inhibitory effect was abolished by miR-421 mimics, demonstrating the protective effects of Rian in BPD mice by targeting miR-421. Furthermore, the secretion of inflammatory cytokines by MLE-12 cells was detected in different groups. The data revealed that TNF- $\alpha$, IL- 6 and IL-1 $\beta$ were markedly upregulated in hyperoxia-induced lung cells. Furthermore, as compared with those in the hyperoxia + control-plasmid group, the TNF- $\alpha$, IL- 6 and IL-1 $\beta$ levels were markedly decreased. However, these effects were abolished by miR-421 mimics. The above findings suggested that Rian has an important role in the development of BPD. However, there were still certain limitations to the present study. For example, in the animal experiment, the rodents were injected continuously whilst being kept under continuous hyperoxia. However, in in vitro experiments, the cells were first subjected to hyperoxia and then transfected with Rian-overexpression vector/miR-421 mimic. The effect of transfecting cells with Rian-overexpression vector/miR-421 mimic under hyperoxia should be further investigated. The mechanisms of BPD include additional pathways apart from the miR-421-Rian axis, which remain to be investigated in the future.

In conclusion, the results of the present study demonstrated that Rian extenuated hyperoxia-induced lung injury in BPD by inhibiting inflammatory response and preventing lung cell apoptosis by targeting miR-421, suggesting that Rian may serve as a potential diagnostic biomarker and therapeutic agent for patients with BPD.

\section{Acknowledgements}

Not applicable.

\section{Funding}

No funding was received.

\section{Availability of data and materials}

The datasets used and/or analyzed during the present study are available from the corresponding author on reasonable request.

\section{Authors' contributions}

XT designed the study, in addition to performing all experiments, analyzing the data and preparing the manuscript. YF 
and $\mathrm{CH}$ contributed to performing the experiments and data collection. All authors read and approved the final manuscript. $\mathrm{XT}$ and YF confirm the authenticity of all the raw data.

\section{Ethics approval and consent to participate}

The present study was approved by the Animal Ethics Committee of the Experimental Animal Center of The First People's Hospital of Lianyungang (Lianyungang, China).

\section{Patient consent for publication}

Not applicable.

\section{Competing interests}

The authors declare that they have no competing interests.

\section{References}

1. Winsper C: The aetiology of borderline personality disorder (BPD): Contemporary theories and putative mechanisms. Curr Opin Psychol 21: 105-110, 2018.

2. Dumpa V and Bhandari V: Surfactant, steroids and non-invasive ventilation in the prevention of BPD. Semin Perinatol 42: 444-452, 2018.

3. Mir IN, Chalak LF, Brown LS, Johnson-Welch S, Heyne R, Rosenfeld CR and Kapadia VS: Impact of multiple placental pathologies on neonatal death, bronchopulmonary dysplasia, and neurodevelopmental impairment in preterm infants. Pediatr Res 87: 885-891, 2020.

4. Maturu P, Wei-Liang Y, Androutsopoulos VP, Jiang W, Wang L, Tsatsakis AM and Couroucli XI: Quercetin attenuates the hyperoxic lung injury in neonatal mice: Implications for Bronchopulmonary dysplasia (BPD). Food Chem Toxicol 114: 23-33, 2018.

5. Bashir RA, Bhandari V, Vayalthrikkovil S, Rabi Y, Soraisham A, Tang S, Al Awad E and Lodha A: Chorioamnionitis at birth does not increase the risk of neurodevelopmental disability in premature infants with bronchopulmonary dysplasia. Acta Paediatr 105: e506-e512, 2016.

6. De Paepe ME, Mao Q, Chao Y, Powell JL, Rubin LP and Sharma S: Hyperoxia-induced apoptosis and Fas/FasL expression in lung epithelial cells. Am J Physiol Lung Cell Mol Physiol 289: L647-L659, 2005

7. Kallapur GS and Jobe AH: Contribution of inflammation to lung injury and development. Arch Dis Child Fetal Neonatal Ed 91: F132-F135, 2006.

8. Fiaturi N, Russo JW, Nielsen HC and Castellot JJ Jr: CCN5 in alveolar epithelial proliferation and differentiation during neonata lung oxygen injury. J Cell Commun Signal 12: 217-229, 2018.

9. Ratner V, Slinko S, Utkina-Sosunova I, Starkov A, Polin RA and Ten VS: Hypoxic stress exacerbates hyperoxia-induced lung injury in a neonatal mouse model of bronchopulmonary dysplasia. Neonatology 95: 299-305, 2009.

10. Zhu Y, Fu J, Yang H, Pan Y, Yao L and Xue X: Hyperoxia-induced methylation decreases RUNX3 in a newborn rat model of bronchopulmonary dysplasia. Respir Res 16: 75, 2015.

11. Mai LJ, Fu XX, He G, Zhao EN and Xue M: Effect of asiaticoside on hyperoxia-induced bronchopulmonary dysplasia in neonatal rats and related mechanism. Zhongguo Dang Dai Er Ke Za Zhi 22: 71-76, 2020 (In Chinese).

12. Hu Y, Xie L, Yu J, Fu H, Zhou D and Liu H: Inhibition of microRNA-29a alleviates hyperoxia-induced bronchopulmonary dysplasia in neonatal mice via upregulation of GAB1. Mol Med 26: 3, 2019.

13. Zhang T, Chen J, Wu H, Pan W, Yang X, Li Y, Liu M and Huang Y: Improved survival and survival without bronchopulmonary dysplasia in very low birth weight infants after active perinatal care. Niger J Clin Pract 23: 980-987, 2020.

14. Salviano-Silva A, Lobo-Alves SC, Almeida RC, Malheiros D and Petzl-Erler ML: Besides pathology: Long non-coding RNA in cell and tissue homeostasis. Noncoding RNA 4: 3, 2018.
15. Bijkerk R, Au YW, Stam W, Duijs JMGJ, Koudijs A, Lievers E, Rabelink TJ and van Zonneveld AJ: Long non-coding RNAs Rian and Miat mediate myofibroblast formation in kidney fibrosis. Front Pharmacol 10: 215, 2019.

16. Gu T, He H, Xing Y, Liu Q, Gu N, Kenkichi S, Jiang H and Wu Q: Expression of non-coding RNA AB063319 derived from Rian gene during mouse development. J Mol Histol 42: 105-112, 2011.

17. Yao P, Li YL, Chen Y, Shen W, Wu KY and Xu WH: Overexpression of long non-coding RNA Rian attenuates cell apoptosis from cerebral ischemia-reperfusion injury via Rian/miR-144-3p/GATA3 signaling. Gene 737: 144411, 2020.

18. Zhong L, Jia J and Ye G: Rian/miR-210-3p/Nfkb1 feedback loop promotes hypoxia-induced cell apoptosis in myocardial infarction through deactivating the PI3K/Akt signaling pathway. J Cardiovasc Pharmacol 76: 207-215, 2020.

19. Liu B, Li J and Cairns MJ: Identifying miRNAs, targets and functions. Brief Bioinform 15: 1-19, 2014.

20. Dupont C, Kappeler L, Saget S, Grandjean V and Levy R: Role of miRNA in the transmission of metabolic diseases associated with paternal diet-induced obesity. Front Genet 10: 337, 2019.

21. Chen J, Wu L, Sun Y, Yin Q, Chen X, Liang S, Meng Q, Long H, Li F, Luo C and Xiao X: Mir-421 in plasma as a potential diagnostic biomarker for precancerous gastric lesions and early gastric cancer. PeerJ 7: e7002, 2019.

22. Ren Z, He M, Shen T, Wang K, Meng Q, Chen X, Zhou L, Han Y, Ji C, Liu S and Fu Q: MiR-421 promotes the development of osteosarcoma by regulating MCPIP1 expression. Cancer Biol Ther 21: 231-240, 2020

23. Yin Y, Xu L, Chang Y, Zeng T, Chen X, Wang A, Groth J, Foo WC, Liang $\mathrm{C}$, Hu $\mathrm{H}$ and Huang J: N-Myc promotes therapeutic resistance development of neuroendocrine prostate cancer by differentially regulating miR-421/ATM pathway. Mol Cancer 18: 11, 2019.

24. Chen L, Tang Y, Wang J, Yan Z and Xu R: miR-421 induces cell proliferation and apoptosis resistance in human nasopharyngeal carcinoma via downregulation of FOXO4. Biochem Biophys Res Commun 435: 745-750, 2013.

25. Yuan HS, Xiong DQ, Huang F, Cui J and Luo H: MicroRNA-421 inhibition alleviates bronchopulmonary dysplasia in a mouse model via targeting Fgf10. J Cell Biochem 120: 16876-16887, 2019.

26. Park MS, Rieger-Fackeldey E, Schanbacher BL, Cook AC, Bauer JA, Rogers LK, Hansen TN, Welty SE and Smith CV: Altered expressions of fibroblast growth factor receptors and alveolarization in neonatal mice exposed to $85 \%$ oxygen. Pediatr Res 62: 652-657, 2007.

27. Livak KJ and Schmittgen TD: Analysis of relative gene expression data using real-time quantitative PCR and the 2(-Delta Delta C(T)) method. Methods 25: 402-408, 2001.

28. Li JH, Liu S, Zhou H, Qu LH and Yang JH: starBase v2.0: Decoding miRNA-ceRNA, miRNA-ncRNA and protein-RNA interaction networks from large-scale CLIP-Seq data. Nucleic Acids Res 42 (Database Issue): D92-D97, 2014.

29. Clément T, Salone V and Rederstorff M: Dual luciferase gene reporter assays to study miRNA function. Methods Mol Biol 1296: 187-198, 2015.

30. Chen X, Zhang X and Pan J: Effect of montelukast on bronchopulmonary dysplasia (BPD) and related mechanisms. Med Sci Monit 25: 1886-1893, 2019.

31. Chen X, Peng W, Zhou R, Zhang Z and Xu J: Montelukast improves bronchopulmonary dysplasia by inhibiting epithelial-mesenchymal transition via inactivating the TGF- $\beta 1 /$ Smads signaling pathway. Mol Med Rep 22: 2564-2572, 2020.

32. Stark A, Dammann C, Nielsen HC and Volpe MV: A pathogenic relationship of bronchopulmonary dysplasia and retinopathy of prematurity? A review of angiogenic mediators in both diseases. Front Pediatr 6: 125, 2018.

33. Kalikkot TR, Guaman MC and Shivanna B: Bronchopulmonary dysplasia: A review of pathogenesis and pathophysiology. Respir Med 132: 170-177, 2017

34. Principi N, Di Pietro GM and Esposito S: Bronchopulmonary dysplasia: Clinical aspects and preventive and therapeutic strategies. J Transl Med 16: 36, 2018.

35. Hwang JS and Rehan VK: Recent advances in bronchopulmonary dysplasia: Pathophysiology, prevention, and treatment. Lung 196: 129-138, 2018

36. van Rossem MC, van de Loo M, Laan BJ, de Sonnaville ES, Tamminga P, van Kaam AH and Onland W: Accuracy of the diagnosis of bronchopulmonary dysplasia in a referral-based health care system. J Pediatr 167: 540-544.e1, 2015. 
37. Abman SH, Collaco JM, Shepherd EG, Keszler M, Cuevas-Guaman M, Welty SE, Truog WE, McGrath-Morrow SA, Moore PE, Rhein LM, et al: Interdisciplinary care of children with severe bronchopulmonary dysplasia. J Pediatr 181: 12-28, 2017.

38. Cai Q, Wang S, Jin L, Weng M, Zhou D, Wang J, Tang Z and Quan Z: Long non-coding RNA GBCDRlncl induces chemoresistance of gallbladder cancer cells by activating autophagy. Mol Cancer 18: 82, 2019

39. Gong X, Qiu J, Qiu G and Cai C: Adrenomedullin regulated by miRNA-574-3p protects premature infants with bronchopulmonary dysplasia. Biosci Rep 40: BSR20191879, 2020.

40. Wang J, Yin J, Wang X, Liu H, Hu Y, Yan X, Zhuang B, Yu Z and Han S: Changing expression profiles of mRNA, lncRNA, circRNA and miRNA in lung tissue reveal the pathophysiological of bronchopulmonary dysplasia (BPD) in mouse model. J Cell Biochem 120: 9369-9380, 2019.

41. Sun Z, Shi K, Yang S, Liu J, Zhou Q, Wang G, Song J, Li Z, Zhang Z and Yuan W: Effect of exosomal miRNA on cancer biology and clinical applications. Mol Cancer 17: 147, 2018.

42. Xu B, Liu J, Xiang X, Liu S, Zhong P, Xie F, Mou T and Lai L: Expression of miRNA-143 in pancreatic cancer and its clinical significance. Cancer Biother Radiopharm 33: 373-379, 2018.
43. Hanna Y, Laliberté C, Ben Fadel N, Lemyre B, Thébaud B, Barrowman N, Bijelic V, Hoey L and Katz SL: Effect of oxygen saturation targets on the incidence of bronchopulmonary dysplasia and duration of respiratory supports in extremely preterm infants. Paediatr Child Health 25: 173-9, 2020.

44. Kaneko M, Sato M, Ogasawara K, Imamura T, Hashimoto K, Momoi N and Hosoya M: Serum cytokine concentrations, chorioamnionitis and the onset of bronchopulmonary dysplasia in premature infants. J Neonatal Perinatal Med 10: 147-155, 2017.

45. Dias-Freitas F, Metelo-Coimbra C and Roncon-Albuquerque RJ: Molecular mechanisms underlying hyperoxia acute lung injury. Respir Med 119: 23-28, 2016.

46. Yin R, Yuan L, Ping L and Hu L: Neonatal bronchopulmonary dysplasia increases neuronal apoptosis in the hippocampus through the HIF-1alpha and p53 pathways. Respir Physiol Neurobiol 220: 81-87, 2016.

(i) (9) This work is licensed under a Creative Commons Attribution-NonCommercial-NoDerivatives 4.0 International (CC BY-NC-ND 4.0) License. 